\title{
Relations between postural sway and cognitive workload during various gaze tasks in healthy young and old people
}

\author{
Miyoung Roh', Euisu Shin', Seungmin Lee ${ }^{2, *}$ \\ 'Frontier Research Institute of Convergence Sports Science, Yonsei University, Seoul, Korea \\ ${ }^{2}$ Department of Physical Education, College of Education, Chungnam National University, Daejeon, Korea
}

The purpose of this study was to determine whether postural control would differ under various gaze tasks while standing in a wide or narrow stance between healthy young and old people, and also investigate whether postural sway and cognitive workload are affected by dualtask balance. Ten young and 10 healthy old people participated in this study. Each participant stood upright under four gaze conditions (fixation, saccade, pursuit, vestibular-ocular reflex) and two stance conditions (wide and narrow stance) in a total of 16 trials. Postural sway was measured by the mean sway amplitude of the center of pressure in the medial-lateral and anteriorposterior directions. Cognitive workload was measured through pupil response as an index of cognitive activity (ICA) by using Eye tracking system and Eyeworks. The results showed that postural sway significantly reduced when performing saccadic eye movement in both groups but greater postural sway was evoked in ves-

\section{INTRODUCTION}

Postural control is the most fundamental movement of various daily activities. It is not a simple, rather a complex system that is affected by various factors such as age, attention, and interaction with sensory information (Woollacott and Shumway-Cook, 2002; Woollacott et al., 1986). Young adults only use a small amount of cognitive demand for postural control, but old people require a large amount of cognitive resources, and this may cause postural instability and falls (Woollacott and Shumway-Cook, 2002). In addition, the visual system plays an important role as an indicator in providing the central nervous system with prior knowledge in order to stabilize the posture (Paquette and Fung, 2011). However, with aging, visual and cognitive functions are impaired and negatively affect postural stability, which increases risks of falls tibular-ocular reflex condition. In addition, although old people had a significant increase in ICA compared to the young, there were no significant differences among all the gaze conditions in old people. These results confirmed that saccadic eye movements are the most beneficial for reducing postural sway regardless of aging and also provide some insight that pupil response represents an indicator of cognitive workload during dual-task balance context. These findings suggest that eye movement exercises may be considered as an effective intervention to improve postural control so a fall prevention program applying eye movement should be extended to individuals who are at risk of falling.

Keywords: Postural control, Eye movements, Cognitive workload, Pupil response, Old people
${ }^{*}$ Corresponding author: Seungmin Lee (iD https://orcid.org/0000-0003-4574-1251 Department of Physical Education, College of Education, Chungnam National University, 99 Daehak-ro, Yuseong-gu, Daejeon 34134, Korea

Email: seungmin@cnu.ac.kr

Received: March 4, 2020 / Accepted: March 22, 2021
(Horak et al., 1989; Salthouse, 2009).

Several studies have been conducted to understand the interaction between postural control and eye movements through behavioral variables (e.g., postural sway). The results showed that saccadic eye movement (SEM), (called rapid eye movements), decreased postural sway compared to fixate on the stationary target in the young adults (Rodrigues et al., 2015; Rougier and Garin, 2007; Stoffregen et al., 2007) and even older adults (Aguiar et al., 2015). On the other hand, pursuit eye movement (PEM), slowly tracking the moving target, has shown contradictory results, either increased (Glasauer et al., 2005; Laurens et al., 2010) or decreased (Rodrigues et al., 2015) postural sway. Besides, in the field of vestibular rehabilitation, oculo-motor and gaze stability exercises (GSEs) have been widely used and proven for improving balance in patients with vestibular dysfunction (Hall et al., 2010) 
and in healthy people (Matsugi et al., 2017; Morimoto et al., 2011; Park, 2017). In particular, Bae (2016) found that performing SEM and PEM only for 5 min improved both plantar sensation and postural control in old people. Despite many studies have been conducted to examine the effects of eye movements, it is unclear which type of eye movements are the most beneficial for improving postural control in old people.

Unfortunately, these effects of eye movement exercise were only assessed through the postural sway variable. Although the positive effects of eye movement on postural control have already been proven in terms of behavioral aspects (Aguiar et al., 2015; Rodrigues et al., 2015; Stoffregen et al., 2007), also measuring cognitive workload with aging would better understand the cognitive involvement of postural control during various gaze tasks. Pupil response is well known as a physiological variable that reflected changes in cognitive load during cognitive tasks (e.g., memory task, auditory Stroop task) (Eckstein et al., 2017), but there is a lack of research during a postural control context. Kahya et al. (2018) reported that challenging balance task with visual occlusion has shown to increase postural sway and cognitive workload in young adults. However, there is a lack of research on how postural control and cognitive workload interact according to eye movements induced by various gaze conditions in old people.

Therefore, the objective of this study was to examine whether postural sway would differ according to various gaze tasks between the young and old people, and also investigate the relations between postural control and cognitive workload when various gaze tasks were performed during standing in a wide or narrow stance. We hypothesize that the SEMs on horizontally moving targets will be confirmed in reducing postural sway the most in old people, as previously observed in young adults (Rodrigues et al., 2015). Specifically, the goal of the study was to clarify how the interaction between postural control and cognitive workload through pupillary response with aging during the dual-task with standing and performing various gaze conditions.

\section{MATERIALS AND METHODS}

\section{Research design}

This study is a mixed factorial design with one between-subjects factor (two age groups) and two within-subjects factors (four gaze conditions and two stance conditions).

\section{Participants}

Ten young adults (22.5 \pm 1.6 years) and ten healthy old people
( $72.9 \pm 1.8$ years) participated in this study. Each group consisted of five men and five women. All participants reported no history of neurological diseases, visual impairment, or balance disorders. Participants were informed about this study and signed written consent form. The protocol of the experiment was approved by the Institutional Review Board of Chungnam National University (201906-SB-076-01).

\section{Apparatus and procedure}

Prior to data collection, participants wore a wireless eye tracker (Dikablis Glasses 3, Ergoneers GmbH, Egling, Bayern, Germany) to record pupil responses at $60 \mathrm{~Hz}$ while performing visual tasks and also monitor whether their eyes were successfully followed visual tasks. The eye-tracking system was calibrated before each trial by the manufacturer's instructions.

Participants were asked to stand upright barefoot on force plate (AMTI-4005, Advanced Mechanical Technology Inc., Watertown, MA, USA) and instructed to perform visual tasks displayed in a monitor $1 \mathrm{~m}$ away from their eye level. They stood under two stance conditions with feet parallel but at the shoulder width (wide stance), or feet together (narrow stance). The visual stimuli were a red dot ( $2 \mathrm{~cm}$ diameter) on a white background generated by the Flash CS4 (Macromedia) and presented on the LCD monitor (LG, 24ML$600 \mathrm{SW}, 1,920 \times 1,080$ pixels, $60 \mathrm{~Hz}$ ). The target appeared on the left and right with a frequency of $0.5 \mathrm{~Hz}$. The visual angle between the left and right targets was $11^{\circ}$ to avoid head movement (Stoffregen et al., 2007). Each participant performed two trials for each of the following gaze conditions; (a) in the fixation condition, participants were asked to fixate on the visual target presented in the center of the monitor under wide or narrow stance condition; (b) in the SEMs condition, participants were asked to follow the target switch with their eyes while standing in a wide or narrow stance. The target started appearing on the left side of the monitor, $9.75 \mathrm{~cm}$ away from the center, then disappearing and reappearing on the right side of the monitor with a frequency of $0.5 \mathrm{~Hz}$; (c) in the PEM condition, participants were asked to pursue a moving target with their eyes while standing in a wide or narrow stance. The target was moving from the left to the right side of the monitor with a frequency of $0.5 \mathrm{~Hz}$; (d) in the vestibular-ocular reflex condition, participants were instructed to move their head from the left to the right with a frequency of $0.5 \mathrm{~Hz}$ (beep sound) while keeping the eyes on the center target of the monitor. Each Participant performed 16 trials ( 4 gaze tasks $\times 2$ stances $\times 2$ trials) presented in random order, each of which lasted $60 \mathrm{sec}$. 


\section{Data analysis}

The main dependent variable was postural sway, which was calculated as the mean sway amplitude of center of pressure (COP) in anteriorposterior (AP) and medial-lateral (ML) directions for each trial. COP value has been shown to be a reliable measurement of postural stability in old people (Bae, 2016). The secondary dependent variable was cognitive workload. Pupil size was measured by an eye-tracking system and computed index of cognitive activity (ICA) by using Eyeworks software (Eyeworks, EyeTracking Inc., Solana Beach, CA, USA). ICA value has a continuous range from 0 (no cognitive workload) to 1 (maximum cognitive workload). ICA value has been used as a physiological variable that revealed changes in cognitive workload (Eckstein et al., 2017). In the present study, we used the maximum ICA value of both eyes in nine young adults excluding one due to the error and ten old people data.

\section{Statistical analysis}

A total of 20 subjects (young group, $n=10$; old group, $n=10$ ) participated in this study. The necessary sample size was calculated based on the minimum of the size $(n=16)$ required through the $G^{*}$ power 3.0.10 program (effect size $=0.25$, power $1-\beta=0.80$, significance level $\alpha=0.05$ ). The Shapiro-Wilk test was used to confirm the distribution of normality of the data. For each dependent variable, three-way analysis of variances (ANOVAs) with repeated measures on the last two factors ( 2 groups $\times 4$ gaze conditions $\times 2$ stances) were used, comparing main effects and their interactions. One-way repeated measures ANOVAs and paired or independent $t$-test were conducted as interactions between two independent variables were significant. Post hoc comparisons were performed through the Bonferonni test when the ANOVAs were significant. Partial eta squared $\left(\eta^{2}\right)$ was analyzed to explain effect

Table 1. Descriptive statistic of the center of pressure $(\mathrm{cm})$ during visual tasks in medial-lateral axis

\begin{tabular}{llcc}
\hline Stance & \multicolumn{1}{c}{ Gaze } & Young group $(\mathrm{n}=10)$ & Old group $(\mathrm{n}=10)$ \\
\hline Wide stance & Fixation & $0.88 \pm 0.32$ & $1.20 \pm 0.41$ \\
& Saccade & $0.68 \pm 0.21$ & $0.98 \pm 0.34$ \\
& Pursuit & $0.69 \pm 0.20$ & $0.96 \pm 0.25$ \\
& Vestibular-ocular reflex & $0.99 \pm 0.31$ & $1.77 \pm 0.43$ \\
Narrow stance & Fixation & $1.90 \pm 0.50$ & $2.31 \pm 0.45$ \\
& Saccade & $1.71 \pm 0.29$ & $2.15 \pm 0.66$ \\
& Pursuit & $2.77 \pm 0.87$ & $2.43 \pm 0.84$ \\
& Vestibular-ocular reflex & $2.53 \pm 0.58$ & $3.42 \pm 0.88$ \\
\hline
\end{tabular}

Values are presented as mean \pm standard deviation. sizes between the independent variable and the dependent variable. IBM SPSS Statistics ver. 25.0 (IBM Co., Armonk, NY, USA) was used for all the analyses and a $P$-value was accepted at a significant level of 0.05 .

\section{RESULTS}

\section{Postural sway during gaze conditions}

Table 1 represents the descriptive statistics of the amplitude of COP during visual tasks between the young and old group in ML direction. Analysis of the ANOVA on the amplitude of COP (Table 2) showed a significant main effect of age $(F[1,18]=9.725, P=$ $0.006)$, gaze $(F[3,54]=19.270, P=0.000)$ and stance $(F[1,18]=$ $218.738, P=0.000)$ in the ML direction. Old people had a significant increase in postural sway compared to the young group, especially during fixation, saccade, and vestibular-ocular condition except for pursuit condition. There was also a significant interaction between age and gaze condition $(F[3,54]=5.256, P=0.003)$, gaze and stance condition $(F[3,54]=7.248, P=0.000)$. All the groups had smaller postural sway during saccade condition compared to other visual conditions. However, old people had greater sway during vestibular-ocular condition. In all visual conditions, there was a greater postural sway while standing narrow than wide stance condition. Mean ratings of the $\mathrm{COP}$ on four gaze tasks between the young and old group in ML and AP directions are presented in Fig. 1.

Table 3 represents the descriptive statistics of the amplitude of COP during visual tasks between the young group and the old group in AP direction. Analysis of the ANOVA on the amplitude of COP (Table 4$)$ showed a significant main effect of gaze $(F[3,54]=$ 9.537, $P=0.000)$ and stance $(F[1,18]=7.688, P=0.013)$, but no main effect of age $(F[1,18]=4.063, P=0.059)$ despite old people

Table 2. Summary of three-way repeated measures analysis of variance of the center of pressure in medial-lateral axis

\begin{tabular}{|c|c|c|c|c|c|c|}
\hline Source & SS & $d f$ & MS & $F$ & $P$ & partial $\eta^{2}$ \\
\hline Group & 5.830 & 1 & 5.830 & $9.725^{* *}$ & 0.006 & 0.351 \\
\hline Gaze & 13.918 & 3 & 4.639 & $19.270^{* * *}$ & 0.000 & 0.517 \\
\hline Group $\times$ gaze & 3.796 & 3 & 1.265 & $5.256^{* *}$ & 0.003 & 0.226 \\
\hline Stance & 75.559 & 1 & 76.559 & $218.738^{* * *}$ & 0.000 & 0.924 \\
\hline Group $\times$ stance & 0.047 & 1 & 0.047 & 0.134 & 0.718 & 0.007 \\
\hline Gaze × stance & 3.751 & 3 & 1.250 & $7.248^{* * *}$ & 0.000 & 0.287 \\
\hline Group $\times$ gaze $\times$ stance & 1.000 & 3 & 0.333 & 1.932 & 0.135 & 0.097 \\
\hline
\end{tabular}

SS, sum of square; MS, mean square; $d f$, degrees of freedom. ${ }^{* *} P<0.01 .{ }^{* *} P<0.001$ 

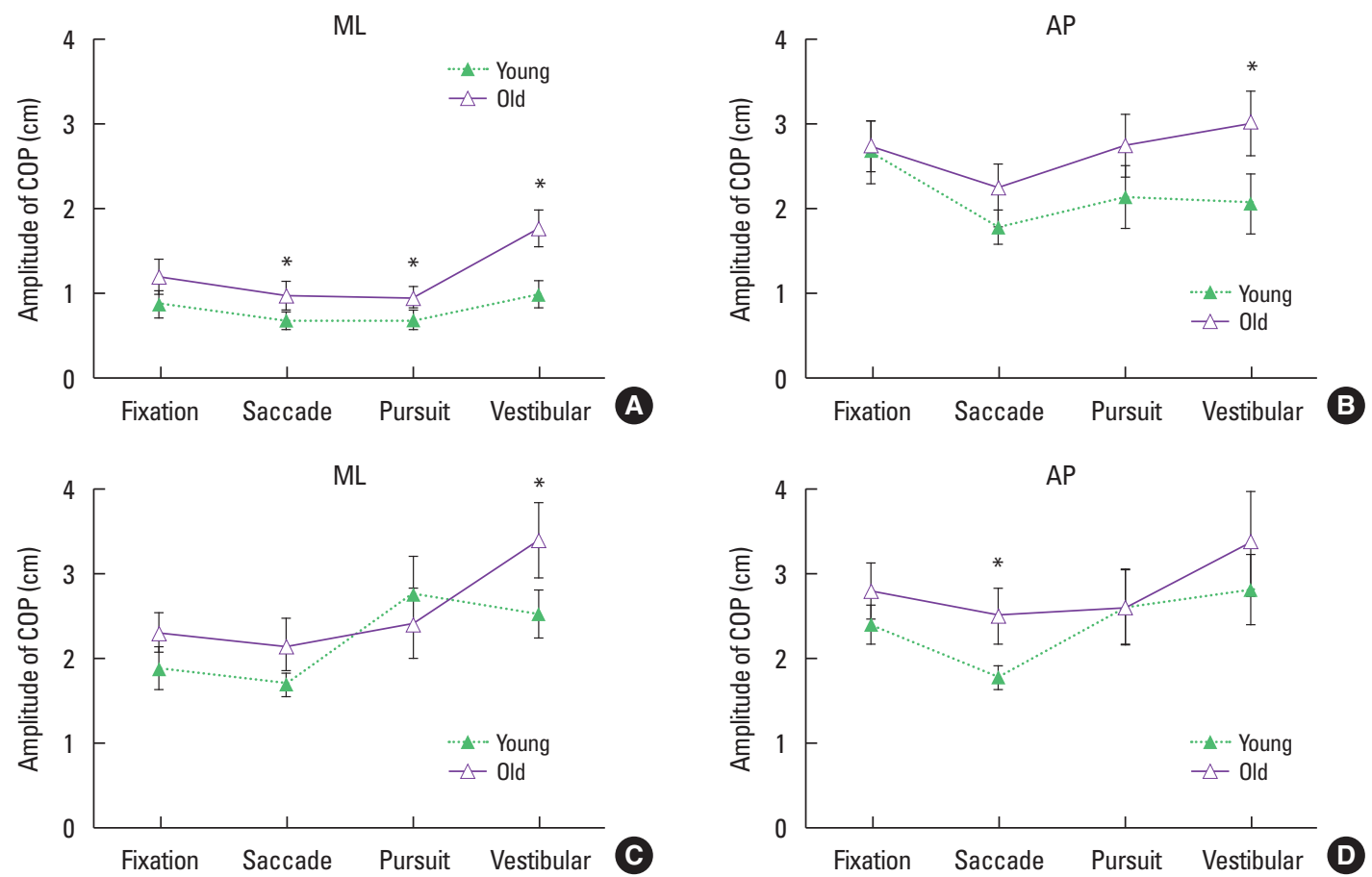

Fig. 1. Mean and standard deviation of amplitude of center of pressure (COP) in medial-lateral (ML) and anteriorposterior (AP) axes for the four gaze conditions (fixation, saccade, pursuit, vestibular= vestibular-ocular reflex) under the wide $(A, B)$ and the narrow $(C, D)$ stance conditions. Error bars depicts the standard deviation. ${ }^{*}$ Significant difference between young and old people during gaze tasks $(P<0.05)$.

Table 3. Descriptive statistics of the center of pressure $(\mathrm{cm})$ during visual tasks in anteriorposterior axis

\begin{tabular}{llcc}
\hline Stance & \multicolumn{1}{c}{ Gaze } & Young group $(n=10)$ & Old group $(n=10)$ \\
\hline Wide stance & Fixation & $2.70 \pm 0.75$ & $2.76 \pm 0.58$ \\
& Saccade & $1.79 \pm 0.42$ & $2.26 \pm 0.58$ \\
& Pursuit & $2.15 \pm 0.75$ & $2.76 \pm 0.76$ \\
& Vestibular-ocular reflex & $2.07 \pm 0.74$ & $3.04 \pm 0.76$ \\
Narrow stance & Fixation & $2.41 \pm 0.46$ & $2.81 \pm 0.68$ \\
& Saccade & $1.78 \pm 0.29$ & $2.51 \pm 0.67$ \\
& Pursuit & $2.61 \pm 0.87$ & $2.61 \pm 0.92$ \\
& Vestibular-ocular reflex & $2.83 \pm 0.83$ & $3.40 \pm 1.15$ \\
\hline
\end{tabular}

Values are presented as mean \pm standard deviation.

$(\mathrm{M}=2.77 \mathrm{~cm})$ had increased postural sway compared with the young $(\mathrm{M}=2.29 \mathrm{~cm})$. There was also interaction between gaze and stance condition $(F[3,54]=4.150, P=0.010)$, among age, gaze, and stance condition $(F[3,54]=2.942, P=0.041)$. Postural sway was greater during vestibular-ocular condition in the narrow stance compared to the wide stance while no differences were observed regarding the other conditions. Postural sway was the smallest during saccade in both stance conditions, but the greatest during fixation condition in the wide stance, and during vestibular-ocular condition in the narrow stance. Additionally, the young had small-
Table 4. Summary of three-way repeated measures analysis of variance of the center of pressure in anteriorposterior axis

\begin{tabular}{lcccccc}
\hline Source & \multicolumn{1}{c}{ SS } & $d f$ & MS & $F$ & $P$ & partial $\eta^{2}$ \\
\hline Group & 9.049 & 1 & 9.049 & 4.063 & 0.059 & 0.184 \\
Gaze & 12.327 & 3 & 4.109 & $9.537^{* * *}$ & 0.000 & 0.346 \\
Group $\times$ gaze & 1.896 & 3 & 0.632 & 1.467 & 0.234 & 0.075 \\
Stance & 1.296 & 1 & 1.296 & $7.688^{*}$ & 0.013 & 0.299 \\
Group $\times$ stance & 0.111 & 1 & 0.111 & 0.660 & 0.427 & 0.035 \\
Gaze $\times$ stance & 2.345 & 3 & 0.782 & $4.150^{*}$ & 0.010 & 0.187 \\
Group $\times$ gaze $\times$ stance & 1.663 & 3 & 0.554 & $2.942^{*}$ & 0.041 & 0.140
\end{tabular}

SS, sum of square; MS, mean square; $d f$, degrees of freedom. ${ }^{*} P<0.05 .{ }^{* *} P<0.001$.

er postural sway in saccade condition compared to the other visual conditions whereas there was greater postural sway when standing a narrow stance compared to the wide stance. However, regardless with stance conditions, old people had smaller postural sway while performing SEMs compared to the fixation and vestibular-ocular reflex condition.

\section{Cognitive workload during gaze conditions}

Table 5 represents the descriptive statistics of the ICA during visual tasks between the young group and the old group. Mean 
Table 5. Descriptive statistics of the index of cognitive activity during visual tasks

\begin{tabular}{llcc}
\hline Stance & \multicolumn{1}{c}{ Gaze } & Young group $(n=9)$ & Old group $(n=10)$ \\
\hline Wide stance & Fixation & $0.12 \pm 0.03$ & $0.31 \pm 0.09$ \\
& Saccade & $0.16 \pm 0.03$ & $0.30 \pm 0.10$ \\
& Pursuit & $0.11 \pm 0.04$ & $0.30 \pm 0.09$ \\
& Vestibular-ocular reflex & $0.23 \pm 0.04$ & $0.32 \pm 0.11$ \\
Narrow stance & Fixation & $0.14 \pm 0.04$ & $0.29 \pm 0.10$ \\
& Saccade & $0.16 \pm 0.04$ & $0.30 \pm 0.11$ \\
& Pursuit & $0.12 \pm 0.03$ & $0.30 \pm 0.10$ \\
& Vestibular-ocular reflex & $0.22 \pm 0.04$ & $0.32 \pm 0.10$ \\
\hline
\end{tabular}

Values are presented as mean \pm standard deviation.

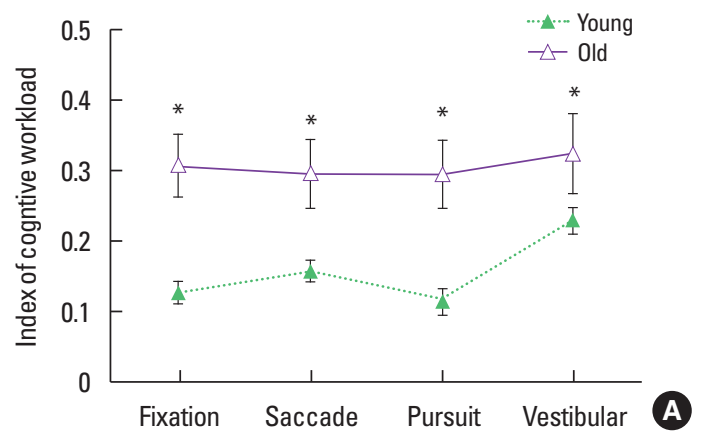

Table 6. Summary of three-way repeated measures analysis of variance of the index of cognitive activity

\begin{tabular}{|c|c|c|c|c|c|c|}
\hline Source & SS & $d f$ & MS & $F$ & $P$ & partial $\eta^{2}$ \\
\hline Group & 0.824 & 1 & 0.824 & $22.139 * * *$ & 0.000 & 0.566 \\
\hline Gaze & 0.096 & 3 & 0.032 & $12.328^{* * *}$ & 0.000 & 0.420 \\
\hline Group $\times$ gaze & 0.045 & 3 & 0.015 & $5.736^{* *}$ & 0.002 & 0.252 \\
\hline Stance & 1.359 & 1 & 1.359 & 0.001 & 0.973 & 0.000 \\
\hline Group $\times$ stance & 0.000 & 1 & 0.000 & 0.241 & 0.630 & 0.014 \\
\hline Gaze×stance & 0.001 & 3 & 0.000 & 0.306 & 0.821 & 0.018 \\
\hline Group $\times$ gaze $\times$ stance & 0.001 & 3 & 0.000 & 0.434 & 0.730 & 0.025 \\
\hline
\end{tabular}

SS, sum of square; $M S$, mean square; $d f$, degrees of freedom. ${ }^{*} P<0.01$. ${ }^{* *} P<0.001$.

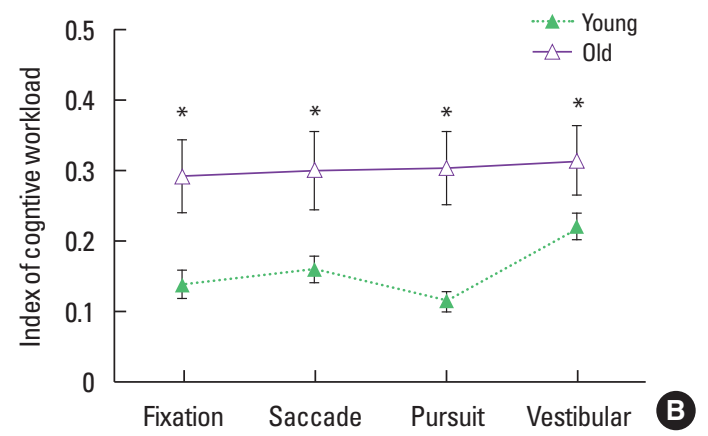

Fig. 2. Mean and standard deviation of the index of cognitive activity in the gaze conditions (fixation, saccade, pursuit, vestibular=vestibular-ocular reflex) under the wide $(\mathrm{A})$ and the narrow $(\mathrm{B})$ stance conditions. Error bars depicts the standard deviation. *Significant difference between young and old people during gaze tasks $(P<0.05)$.

ratings of ICA on four gaze tasks between the young and old group are presented in Fig. 2. Analysis of the ANOVA on cognitive workload, ICA (Table 6$)$ revealed a significant main effect of age $(F[1,17]=$ $22.139, P=0.000)$ and gaze condition $(F[3,51]=12.328, P=0.000)$ but no significant effect of stance condition. Old people had a significant increase in ICA values compared to the young group. In the vestibular-ocular condition, ICA values were significantly increased compared to the other conditions. There was also interaction between age and gaze condition $(F[3,51]=5.736, P=0.002)$. Old people had a significant increase in ICA values during all the gaze conditions compared to the young group $(P<0.05)$. Interestingly, the young had a significant increase in ICA values during the vestibular-ocular condition compared to the other conditions while there were no significant differences among all the gaze conditions in old people.

\section{DISCUSSION}

The effects of eye movement exercises have been proven to improve postural control in previous studies. However, it is unclear which type of eye movements are the most beneficial for reducing postural sway in old people. Thus, the aim of this study was to examine whether postural sway would differ according to various gaze tasks; fixation, saccade, pursuit, vestibular-ocular reflex between young and old people, and also investigate whether postural sway and cognitive workload are affected by gaze tasks while standing in a wide or narrow stance. We assumed that the SEMs will be confirmed in reducing postural sway the most in old people, as previously observed in the young adults (Rodrigues et al., 2015).

The results of this study support our hypothesis that postural sway is significantly reduced when performing SEMs compared the other visual conditions in the young but also in old people. These results are consistent with the fact that SEM leads to a greater postural improvement compared to looking at a stationary target (Aguiar et al., 2015; Giveans et al., 2011; Rodrigues et al., 2015; Stoffregen et al., 2007). The possible mechanism of reducing postural sway in old people can be explained by the evidence of functional integration of postural control with gaze performance (Stoffregen et al., 2007) that posture can be controlled for facilitating the spatial accuracy of eye movement (Kowler, 2011) while 
performing visually guided gaze tasks.

Aguiar et al. (2015) only focused on the effect of SEMs on postural control in old people while standing in a wide or narrow stance as the difficulty of the postural task but the present study examined the influence of various gaze tasks on postural sway with aging. We found that there was greater postural sway during the vestibular-ocular reflex condition in both groups, suggesting that the vestibular-ocular reflex task [including head motion] may cause postural sway, unlike the other gaze tasks. On the other hand, regarding stance conditions, different postural control strategies were adapted between the two groups in that the young had larger sways under a narrow stance compared with a wide stance but old people were not affected by stance conditions. This result supports the finding of the previous study (Aguiar et al., 2015) that old people adopted a more rigid body orientation, not allowing greater sway, under a more difficult stance condition compared with young adults in order to successfully perform the supra-postural task such as SEMs. The reason for old people to adopt a rigid postural strategy could be that old people have limited attentional resources compared with young adults (Woollacott and Shumway-Cook, 2002) so old people accept the gaze task as a more difficult task and thus require greater postural stability, and led to this strategy.

To our knowledge, this is the first study to examine and measure postural sway and also cognitive workload during a dual-task balance context in old people. We found that old people group had a greater cognitive workload compared to the young group having a significant increase in ICA values during the vestibularocular reflex condition whereas there were no significant differences among all the gaze conditions in old people. These results were partially in line with the evidence that the challenging gaze task is associated with greater cognitive workload during dualtask balance, as observed in healthy young adults (Kahya et al., 2018). Vestibular-ocular reflex, called GSE, has already been proven as an effective intervention for improving balance for individuals with healthy old people (Bae, 2016; Bhardwaj and Vats, 2014; Roh and Lee, 2019) and vestibular impairment (Hall et al., 2010; Horning and Gorman, 2007) but only found after GSE intervention setting. Interestingly, in the present study, we examined whether postural control is affected during GSE setting, as a result of standing and concurrently performing the vestibular-ocular reflex was likely to be a challenging task and increased both cognitive workload and postural sway as well. These findings suggest that researchers and practitioners in the field of vestibular rehabilitation have to be cautious when applying GSE for individuals who are at risk of falling.
Pupillometry has been widely used to measure cognitive workload during cognitive tasks (Eckstein et al., 2017) but there was a lack of such research during postural control context in old people. Thus, although the results of this study are not able to directly compare with the previous studies, findings that were no main effect of gaze condition on cognitive workload unlike findings of postural control in old people, it might be due to within-subject experimental design. The possible explanation contributing to greater cognitive workload in old people regardless of various gaze tasks could be random order in tasks of the within-subject experimental design, indicating more frequently rests requested thus it seemed to be hard to detect the differences in cognitive workload in accordance with different gaze tasks.

In conclusion, the present study demonstrated that performing SEM is the most beneficial to reduce postural sway during dualtask balance in both the young and old people even while standing in a narrow stance but vestibular-ocular reflex was evoked greater postural sway. In addition, old people had a significant increase in ICA compared to the young whereas we were not able to determine the certainty of interaction between postural control and cognitive workload during various gaze tasks. Nevertheless, this study contributes to researchers and practitioners in exercise rehabilitation to provide some insight that pupil response represents as an indicator of cognitive workload during dual-task balance context. Therefore, researchers and practitioners in the field of exercise rehabilitation should consider including eye movement exercises when developing or applying fall prevention programs for old people and in future research, they can use pupil response as an effective assessment compared to other expensive neurophysiological tools, such as functional magnetic resonance imaging or electroencephalography to investigate cognitive workload during the dual-task balance. Further studies are needed to clarify the influence of different types of gaze tasks during dual-task balance in old people, suggesting a between-subject design that divides into groups of each eye movement not including the mixed effects of each eye movement.

\section{CONFLICT OF INTEREST}

No potential conflict of interest relevant to this article was reported.

\section{ACKNOWLEDGMENTS}

This study was supported by the National Research Foundation 
of Korea Grant funded by the Korean Government (NRF-2018S1A5B5A01039578).

\section{REFERENCES}

Aguiar SA, Polastri PF, Godoi D, Moraes R, Barela JA, Rodrigues ST. Effects of saccadic eye movements on postural control in older adults. Psychol Neurosci 2015;8:19-27.

Bae Y. Saccadic eye movement improves plantar sensation and postural balance in elderly women. Tohoku J Exp Med 2016;239:159-164.

Bhardwaj V, Vats M. Effectiveness of gaze stability exercise on balance in healthy elderly population. Int J Physiother Res 2014;2:642-647.

Eckstein MK, Guerra-Carrillo B, Singley ATM, Bunge SA. Beyond eye gaze: what else can eyetracking reveal about cognition and cognitive development? Dev Cogn Neurosci 2017;25:69-91.

Giveans MR, Yoshida K, Bardy B, Riley M, Stoffregen TA. Postural sway and the amplitude of horizontal eye movements. Ecol Pshychol 2011; 23:247-266.

Glasauer S, Schneider E, Jahn K, Strupp M, Brandt T. How the eyes move the body. Neurology 2005;65:1291-1293.

Hall CD, Heusel-Gillig L, Tusa RJ, Herdman SJ. Efficacy of gaze stability exercises in older adults with dizziness. J Neurol Phys Ther 2010;34: 64-69.

Horak FB, Shupert CL, Mirka A. Components of postural dyscontrol in the elderly: a review. Neurobiol Aging 1989;10:727-738.

Horning E, Gorman SL. Vestibular rehabilitation decreases fall risk and improves gaze stability for an older individual with unilateral vestibular hypofunction. J Geriatr Phys Ther 2007;30:121-127.

Kahya M, Wood TA, Sosnoff JJ, Devos H. Increased postural demand is associated with greater cognitive workload in healthy young adults: a pupillometry study. Front Hum Neurosci 2018;12:288.

Kowler E. Eye movements: the past 25 years. Vision Res 2011;51:1457-1483.

Laurens J, Awai L, Bockisch C, Hegemann S, Van Hedel H, Dietz V, Straumann D. Visual contribution to postural stability: Interaction between target fixation or tracking and static or dynamic large-field stimulus. Gait Posture 2010;31:37-41.

Matsugi A, Ueta Y, Oku K, Okuno K, Tamaru Y, Nomura S, Tanaka H, Mori N. Effect of gaze-stabilization exercises on vestibular function during postural control. Neuroreport 2017;28:439-443.

Morimoto H, Asai Y, Johnson EG, Lohman EB, Khoo K, Mizutani Y, Mizutani T. Effect of oculo-motor and gaze stability exercises on postural stability and dynamic visual acuity in healthy young adults. Gait Posture 2011;33:600-603.

Paquette C, Fung J. Old age affects gaze and postural coordination. Gait Posture 2011;33:227-232.

Park J. The effects of eyeball exercise on balance ability and falls efficacy of the elderly who have experienced a fall: a single-blind, randomized controlled trial. Arch Gerontol Geriatr 2017;68:181-185.

Rodrigues ST, Polastri PF, Carvalho JC, Barela JA, Moraes R, Barbieri FA. Saccadic and smooth pursuit eye movements attenuate postural sway similarly. Neurosci Lett 2015;584:292-295.

Roh M, Lee E. Effects of gaze stability exercises on cognitive function, dynamic postural ability, balance confidence, and subjective health status in old people with mild cognitive impairment. J Exerc Rehabil 2019;15: 270-274.

Rougier P, Garin M. Performing saccadic eye movements or blinking improves postural control. Motor Control 2007;11:213-223.

Salthouse TA. When does age-related cognitive decline begin? Neurobiol Aging 2009;30:507-514.

Stoffregen TA, Bardy BG, Bonnet CT, Hove P, Oullier O. Postural sway and the frequency of horizontal eye movements. Motor Control 2007; 11:86-102.

Woollacott M, Shumway-Cook A. Attention and the control of posture and gait: a review of an emerging area of research. Gait Posture 2002; 16:1-14.

Woollacott MH, Shumway-Cook A, Nashner LM. Aging and posture control: changes in sensory organization and muscular coordination. Int J Aging Hum Dev 1986;23:97-114. 